\title{
A Smart Wheelchair Based on Gesture Control and Vital Signs Monitoring
}

\author{
Miao Chi \\ Automation School \\ Beijing University of Posts and Telecommunications \\ Beijing, China \\ chimiao123123@163.com
}

\begin{abstract}
Aiming to offer better ubiquitous healthcare (Uhealthcare) to the aged and the disabled, this paper introduce a smart wheelchair with embedded sensor system for vital signs which can be controlled by simple gestures. In motion control module, firstly the gesture is distinguished from background using depth images from Kinect. Then three detailed formulas are added to $\mathrm{Hu}$ Invariant Moments for hand gesture recognition. Finally, recognition results are transmitted to a host computer via USB 2.0 interface for implementing motion control. In sensor sub-system module, a microcontroller-based platform is designed to process the signals required from biomedical sensors embedded in the wheelchair and output signals are transmitted wirelessly using IEEE802.15.4 WPAN protocol to a server application implemented in a host computer. Physiological parameters are measured by photoplethysmography (PPG), ballistocardiography (BCG) and skin conductivity sensor. Experimental results and analyses show that such a smart wheelchair can provide the aged and disabled people with better U-healthcare.
\end{abstract}

Keywords-U-healthcare; smart biosensors; IEEE1451 standard; Hu Invariant Moment; intelligent wheelchair.

\section{INTRODUCTION}

At present, considering the fact that the proportion of the aged and disabled people has increased in the worldwide, it is necessary to promote U-healthcare development for them [1]. Many researchers have paid attentions to the smart wheelchair which can offer independent mobility and vital signs monitoring to elderly and disabled people.

In the field of wheelchair motion control, people could not control the wheelchair properly in some cases, because most wheelchairs are too heavy [2]. A research entitled "Kinectbased Powered Wheelchair Control System" has been done by C. Chang [3], providing a design of hand gesture recognition with Kinect interface. Another study by V. Megavannan [4], entitled "Human Action Recognition using Depth Maps" provides the usage of the scale, translation and rotation invariant $\mathrm{Hu}$ moments to represent the features of the depth image from Kinect.

In the field of vital signs monitoring, photoplethysmography (PPG), ballistocardiography (BCG) and skin conductivity sensor are widely used to measure the physical condition of the aged and the disabled [5]. Instead of an enduring in-hospital cure needed by the seriously sick people, the elderly and disabled people with certain capacity to arrange their life and activities only need some long-term monitoring $[6,7]$. Besides, no medical interference is required until disturbance emerges in their physiological parameters [8]. Aiming to assure the modularity of smart wheelchair system, the vital signs sensors can be assembled in the system as plugand-play modules [9-11]. A research conducted by $\mathrm{O}$. Postolache, entitled "Smart walker for pervasive healthcare" [12] also provide a developed prototype in the field of smart objects.

Based on the researches available so far, a smart wheelchair which includes two modules for simplifying motion control method and monitoring vital signs of wheelchair users is proposed in this paper. On the basis of Hu Invariant Moments, three detailed formulas are added for hand gesture recognition in motion control module. The sensor sub-system module is composed of a microcontroller-based platform, an external connection board, a sensor interface board and plug-and-play biomedical sensors. The physicians can extract information about wheelchair users' health status through the analyses on physiological parameters got from biomedical sensors and then work out the corresponding treatment for chronic disease prevention.

This paper is organized as follows: In Chapter I, we present the introduction to present the background information. In Chapter II, the motion control module of the smart wheelchair system is described. The sensor sub-system module is introduced in Section III. Section IV shows the experimental results and analyses. Finally we make a brief conclusion in Section V. 


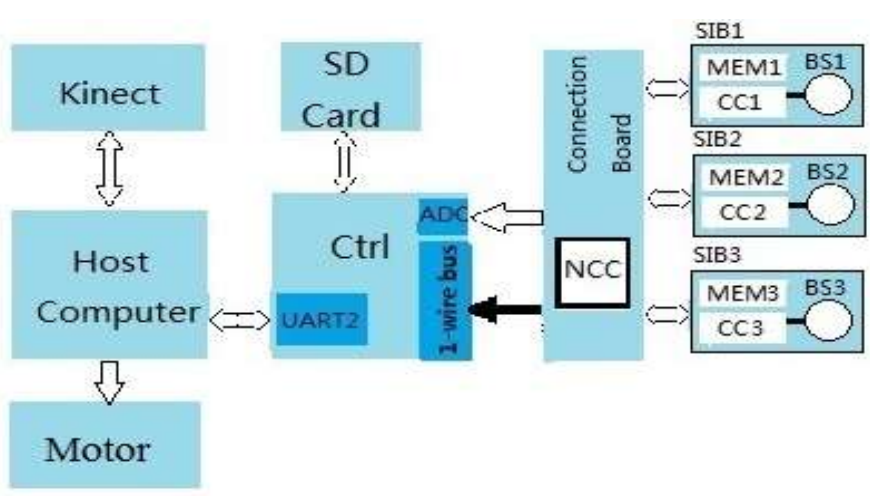

Figure 1.The block diagram of a smart wheelchair system (BS1photoplethysmography sensor, BS2-ballistocardiography sensor, BS1-skin conductivity sensor, MEM- 1-wire EEPROM, CCi-conditioning circuit, NCCnetwork control circuit)

\section{Motion CONTROL MODULE}

\section{A. Image Depth Information Acquisition}

The image depth information is acquired by the collaboration of an infrared transmitter and an infrared CMOS camera embedded in Kinect which is transmitted to PS1080SoC chip for producing a depth image of the scene. Since all algorithms of depth information acquisition run on the PS1080SoC chip, the host computer only need to receive processed data based on a USB 2.0 physical layer protocol.

\section{B. Gesture Recongnition Based on Hu Invariant Moments}

HU Invariant Moment [13] is widely used since 1962 due to its proportion, translation and rotation invariance. Considering the image gray value as a two-dimensional or three-dimensional density distribution function $\mathrm{f}(\mathrm{x}, \mathrm{y}), \mathrm{Hu}$ Invariant Moments is used to extract image feature in the field of image analysis. Two-dimensional $(p+q)$ order moment in the discrete case is defined as follows:

$$
m_{p q}=\sum_{x=1}^{M} \sum_{y=1}^{N} x^{p} y^{q} f(\mathrm{x}, \mathrm{y}) \quad p, q=0,1,2 \ldots
$$

The central moment is defined as:

$$
\mu_{p q}=\sum_{x=1}^{M} \sum_{y=1}^{N}(x-\bar{x})^{p}(y-\bar{y})^{q} f(\mathrm{x}, \mathrm{y}),
$$

where

$$
\bar{x}=m_{10} / m_{00}, \bar{y}=m_{01} / m_{00} .
$$

According to all definition above, the seven invariant moments are as follows:

$$
\begin{aligned}
& \Phi_{1}=\eta_{20}+\eta_{02} \\
& \Phi_{2}=\left(\eta_{20}-\eta_{02}\right)^{2}+4 \eta_{11}^{2} \\
& \Phi_{3}=\left(\eta_{30}-3 \eta_{12}\right)^{2}+\left(3 \eta_{21}-\eta_{03}\right)^{2} \\
& \Phi_{4}=\left(\eta_{30}+\eta_{12}\right)^{2}+\left(\eta_{21}+\eta_{03}\right)^{2}
\end{aligned}
$$

$$
\begin{aligned}
& \Phi_{5}=\left[\left(\eta_{30}+\eta_{12}\right)^{2}-3\left(\eta_{21}+\eta_{03}\right)^{2}\right]\left(\eta_{30}-3 \eta_{12}\right)\left(\eta_{12}+\eta_{30}\right) \\
& +\left[3\left(\eta_{30}+\eta_{12}\right)^{2}-\left(\eta_{21}+\eta_{03}\right)^{2}\right]\left(3 \eta_{21}-\eta_{03}\right)\left(\eta_{21}+\eta_{03}\right) \\
& \Phi_{6}=\left[\left(\eta_{30}+\eta_{02}\right)^{2}-\left(\eta_{21}+\eta_{03}\right)^{2}\right]\left(\eta_{20}-\eta_{02}\right) \\
& +4 \eta_{11}\left(\eta_{30}-\eta_{12}\right)\left(\eta_{21}+\eta_{03}\right) \\
& \Phi_{7}=\left(3 \eta_{21}-\eta_{30}\right)\left(\eta_{30}+\eta_{12}\right)\left[\left(\eta_{30}+\eta_{12}\right)^{2}-3\left(\eta_{21}+\eta_{03}\right)^{2}\right] \\
& -\left[3\left(\eta_{30}+\eta_{12}\right)^{2}-\left(\eta_{21}+\eta_{02}\right)^{2}\right]\left(\eta_{30}-3 \eta_{12}\right)\left(\eta_{21}+\eta_{03}\right)
\end{aligned}
$$

Aiming at accurate gesture recognition, three low order formulas based on $\mathrm{Hu}$ Invariant Moment are expressed as follows:

$$
\begin{aligned}
& \Phi_{8}=2 \eta_{11}\left[\left(\eta_{30}+\eta_{12}\right)^{2}-\left(\eta_{21}+\eta_{08}\right)^{2}\right] \\
& -2\left(\eta_{20}-\eta_{02}\right)\left(\eta_{30}+\eta_{12}\right)\left(\eta_{21}+\eta_{08}\right) \\
& \Phi_{9}=\left[\left(\eta_{30}-3 \eta_{12}\right)\left(\eta_{30}+\eta_{12}\right)+\left(3 \eta_{21}+\eta_{03}\right)\left(\eta_{21}+\eta_{03}\right)\right]\left(\eta_{20}+\eta_{03}\right) \\
& +2 \eta_{11}\left[3\left(\eta_{21}+\eta_{03}\right)\left(\eta_{30}+\eta_{12}\right)-\left(\eta_{30}-3 \eta_{12}\right)\left(\eta_{21}+\eta_{03}\right)\right]
\end{aligned}
$$

$\Phi_{10}=\left[\left(3 \eta_{21}-\eta_{08}\right)\left(\eta_{30}+\eta_{12}\right)-\left(\eta_{30}-3 \eta_{12}\right)\left(\eta_{21}+\eta_{03}\right)\right]\left(\eta_{20}+\eta_{02}\right)$

$-2 \eta_{11}\left[\left(\eta_{30}-3 \eta_{12}\right)\left(\eta_{30}+\eta_{12}\right)+\left(3 \eta_{21}-\eta_{03}\right)\left(\eta_{21}+\eta_{03}\right)\right]$

Five gestures with discrimination shown in Fig .2 from left to right are selected as wheelchair motion control commands which are for: forward, back, left, right and stop.

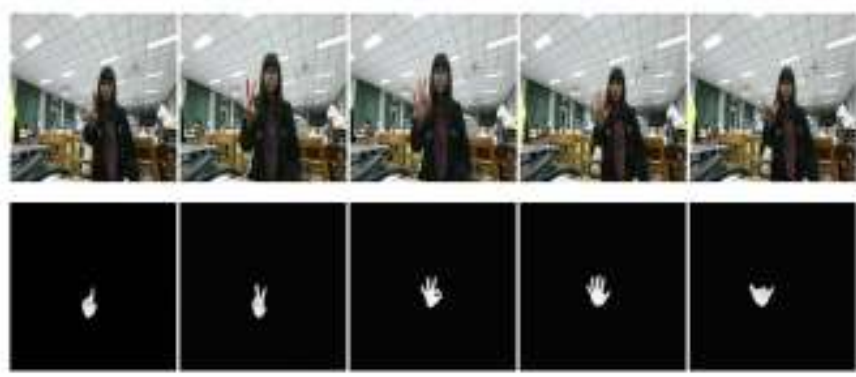

Figure 2. Hand gesture templates

Verified by experiment, gestures can be easily distinguished from the background using the above algorithm for further process.

\section{Motion Control System of Smart Wheelchair}

As shown in Fig .3 the process of controlling the wheelchair movement includes four steps.

Step1: Obtain the depth information image from the normal image by Kinect

Step2: Convert the depth information image into the gesture binary image based on $\mathrm{Hu}$ Invariant Moments

Step3: The gesture binary image is recognized and converted into the corresponding motion control command

Step4: Control the movement of the wheelchair using the instruction issued by the host computer 


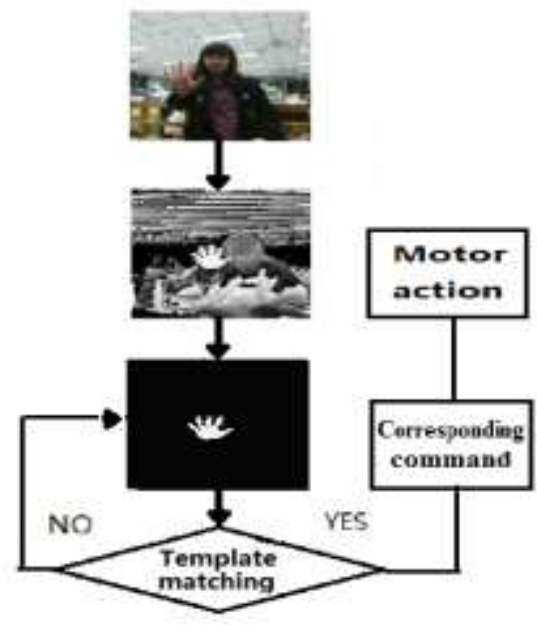

Figure 3. System flow chart

\section{SENSOR SUB-SYSTEM MODULE}

\section{A. Microcontroller-based Platform}

The microcontroller-based platform [14] consists of a PIC 24F128GA010 microcontroller, a 10-bit A/D converter, a 1wire bus interface, and a Universal Asynchronous Receiver/Transmitter (UART) interface, which is responsible to manage biomedical sensors and communicate with host computer. Specifications of these objects under operation are as follows:

- The microcontroller operates at a frequency of $8 \mathrm{MHz}$

- The 10-bit A/D converter sampling rate can reach to maximum at $10 \mathrm{kS} / \mathrm{s}$

- The conditioning circuit must qualified signal amplitude change within $3.3 \mathrm{~V}$

- The digital interface is responsible to transmit all the multi-drop bus information using 1-wire bus

- The function of the UART interface is to communicate with the host computer

\section{B. External Connection Board}

The external connection board between microcontroller board and sensor interface board makes the system modular because sensor output connectors are not directly attached to the microcontroller-based board. The usage of analog multiplexer CD4051BF embedded in the connection board to integrate 8 single point-point connections into a 1-wire multidrop bus reduces the digital ports requirement of microcontroller.

The joint between the microcontroller-based platform and the connection board is a 20-pin cable. Additionally, the association between sensor interface board and the connection board is made via a 5-pin connector, which has pins for $5 \mathrm{~V}$, $3.3 \mathrm{~V}$, analog signal, digital data and ground.

\section{Sensor Interface Board and Biomedical Sensors}

All the transducers were designed to satisfy the IEEE 1451.4 MMI Class 2 specifications making the system more reliable. Each sensor interface board is attached to an analog biomedical sensor as a node of the smart sensor network. The main component of the sensor interface board is the 1-wire EEPROM: DS24B33 with 1kbytes of memory to store 64-bit digital information based on IEEE 1451.4 Basic TEDS. As the Fig .4 shows the biomedical sensors, 1-wire EEPRPM and conditioning circuit are powered by $3.3 \mathrm{~V}$ and $5 \mathrm{~V}$.

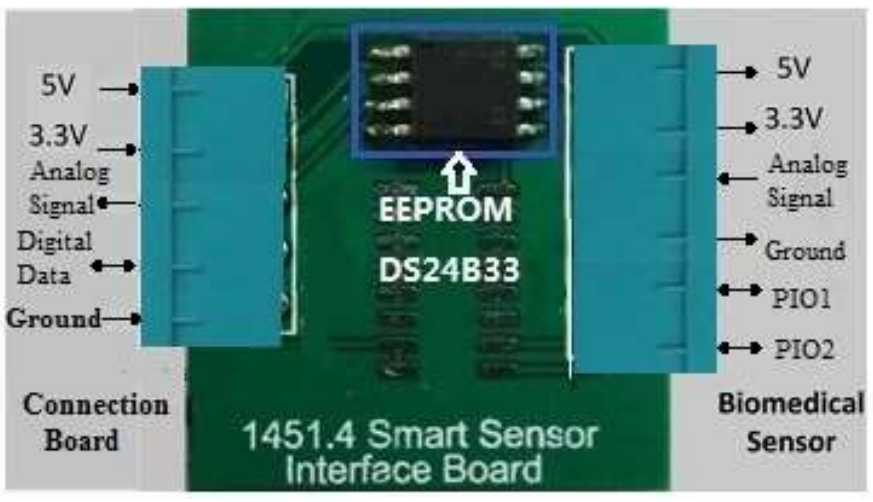

Figure 4. Smart Sensor Interface Board

The cardiorespiratory assessment is performed by photoplethysmography ( PPG ) , aballistocardiography

(BCG), and a skin conductivity sensor. The $\mathrm{SpO} 2$ sensor is used to obtain PPG signal while electromechanical film ( EMFi ) for BCG signal. As one of vital signs, diastolic blood pressure and systolic blood pressure can be estimated by calculating the time delay from the BCG peak to the corresponding PPG peak. But this measurement method has a drawback in estimating the BCG peak locations, which will be described further in chapter IV.

\section{EXPERIMENTAL RESULTS AND ANALYSES}

The experimental results and analyses are separated into two parts, gesture recognition based on Hu Invariant Moments and availability verification of wheelchair system parts.

\section{A. Experimental Results and Analyses of Gesture Recognition}

1) $\mathrm{Hu}$ moments characteristics verification of scale, translation and rotation

We apply $\mathrm{Hu}$ Invariant Moments to the image matching based on its characteristics of scale, translation and rotation. Some experimental results of $\mathrm{Hu}$ moments characteristics verification are shown in Fig .5. 


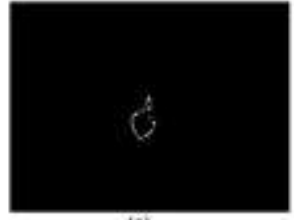

(a)

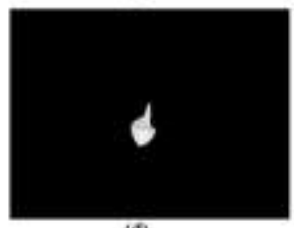

(4)
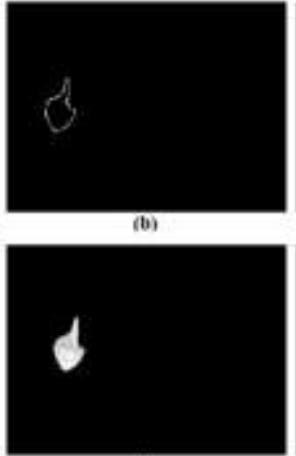

(c)

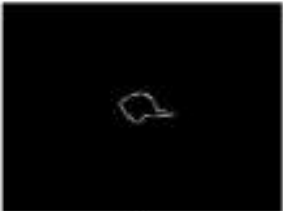

$(c)$

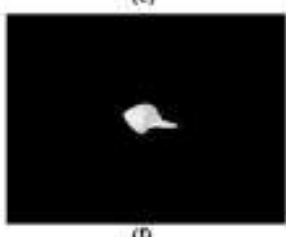

(1)
Figure 5. Triple invariant patterns of $\mathrm{Hu}$ moments. Gesture template under scale (a), translation (b) and rotation (c). Gesture recognition under scale (d), translation (e) and rotation (f).

Since the triple invariant patterns of $\mathrm{Hu}$ moments have been improved, we can reach the conclusion: Gesture can be recognized when it changes by scaling, translating and rotating.

\section{2) Robustness verification of gesture recognition.}

Experiments under different conditions have been done to verify the robustness of gesture recognition. The robustness verification is accomplished under three conditions: weak light (a), strong light (b) and interferential background (c).
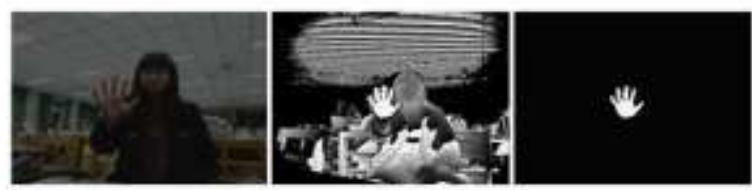

(a)

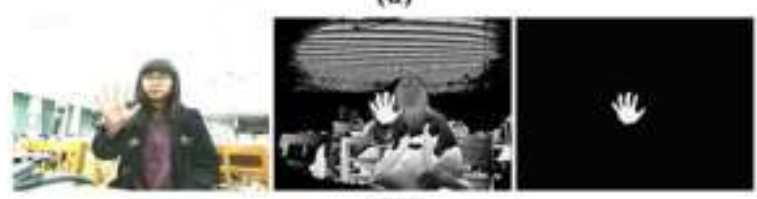

(b)
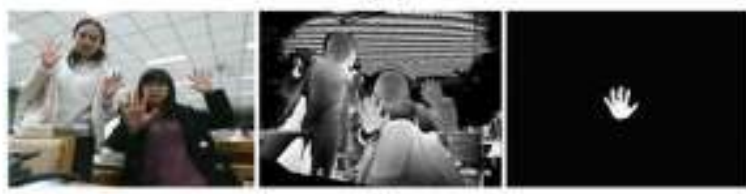

(c)

Figure 6. Gesture recognition under different conditions

As shown in Fig .6 the experiment results of robustness verification prove that our system can be applied under different conditions

\section{B. Availability Verification of Wheelchair System}

\section{1) Assessment of wheelchair's movement.}

We choose three aged people who are not familiar with our system to control the smart wheelchair in the designed trajectory (Fig .7).

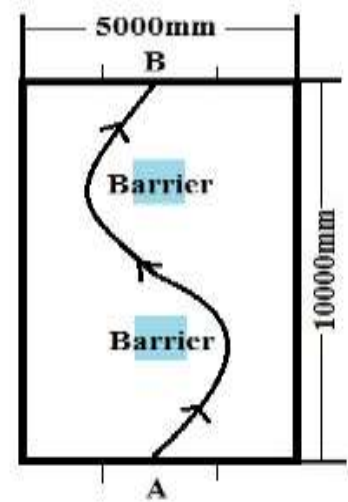

Figure 7. Wheelchair roadmap

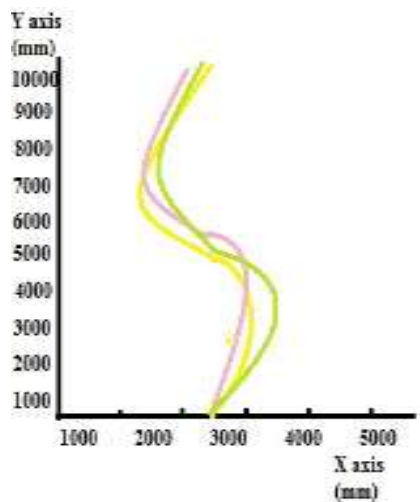

Figure 8. Experimental trajectories
In Fig .8 three different colored lines represent experimental trajectories made by three aged individuals.

The experiment result displayed in Fig .8 proves that the smart wheelchair system controlled by simple gesture performs satisfactorily.

2) Vital signs measurement based on biomedical sensors

In the smart wheelchair system, PPG, BCG and skin conductivity sensor are used to monitor vital signs of wheelchair user.

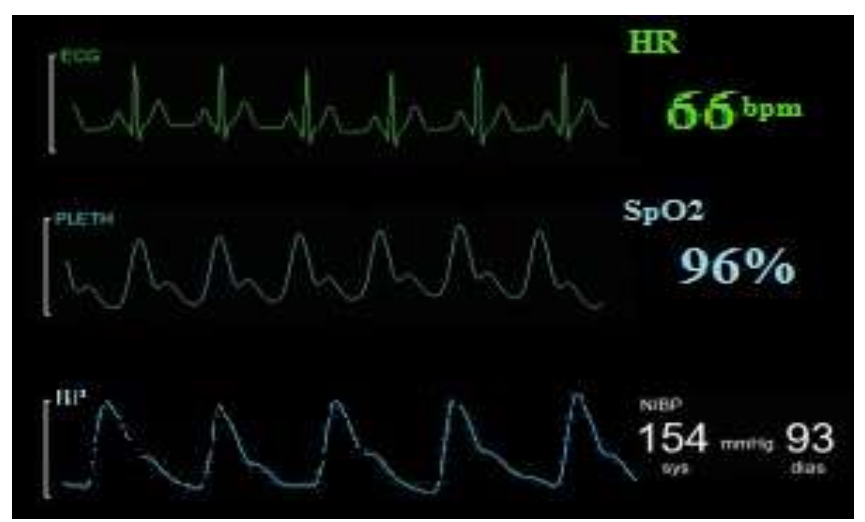

Figure 9. Vital signs measurement

There are some weaknesses verified by vital signs measurement. For example motion noises have a negative influence in estimating the BCG peak locations. Further investigation is needed to reduce the interference of wheelchair motion for assuring the system accuracy.

\section{CONCLUSION}

The design of an intelligent wheelchair with embedded sensor system for vital signs which can be controlled by simple gesture is introduced in this paper. A particular description of using $\mathrm{Hu}$ Invariant Moments for hand gesture recognition based on the depth images from Kinect was made. Additionally the high modularity and extensibility of the sensor system architecture are due to the adoption of the IEEE1451.4 standards. Further researches of monitoring more vital signs and wheelchair outdoor navigation are needed to realize ubiquitous healthcare for the aged and disabled people. 


\section{REFERENCES}

[1] K. Dhama, S. Chakraborty, S. Kapoor et al., "One world,one healthveterinary perspectives," Advances in Animal and Veterinary Sciences, vol. 1, no. 1, pp. 5-13, 2013.

[2] Y. T. Wang, R. Bernard, C. Cope, L. S. Chang, W. Limroongreungrat and S. Sprigle, "Fundamental locomotive activity time efficiency with differently positioning drive-axis wheelchairs among elders." Adapt Phys Activ Q, vol. 25, no. 4, pp. 322-334, 2008.

[3] C. L. Chang, C. C. Chen, C. Y. Chen and B. S. Lin, " Kinect-based Powered Wheelchair Control System." IEEE 2013 4th International Conference on Intelligent Systems Modelling and Simulation, pp. 186189, 2013.

[4] V. Megavannan, B. Agarwal and R.Venkatesh Babu, "Human action recognition using depth maps." IEEE Signal Processing and Communications (SPCOM) International Conference, pp. 1-5, July 2012.

[5] T F. Budinger, "Biomonitoring with wireless communications." Annual Review of Biomedical Engineering, vol. 5, no. 1, pp. 383-412,2003

[6] T. Tanaka, K. Sonoda, S. Okochi et al., "Wearable health monitoring system and its applications." International Conference on Emerging Trends in Engineering \& Technology, pp. 143-146. 2011.

[7] L. F. Purwoko, Y. Priyana and T. Mardiono, "Ubiquitous Health Monitoring System design." 2013 Joint International Conference on Rural Information \& Communication Technology and Electric-Vehicle Technology, pp. 1-6. 2013.
[8] Y. Wang, X. Hu, "Fuzzy Reasoning of Accident Provenance in Pervasive Healthcare Monitoring Systems", IEEE Journal of Biomedical and Health Informatics, Vol. 17, No. 6, pp. 1015-1022, Novermber, 2013.

[9] O. Postolache, P. Girão, J.M. Joaquim, E. Pinheiro, G. Postolache; "Physiological Parameters Measurement Based on Wheelchair Embedded Sensors and Advanced Signal Processing", IEEE Trans. on Instrumentation and Measurement, Vol. 59, No. 10, pp. 2564 - 2574, October, 2010

[10] P. Doyle, D. Heffernan, D. Duma, “A time-triggered transducer network based on an enhanced IEEE 1451 model", Microprocess. Microsyst. 28 (2004) 1-12

[11] P. A. Neves, B. Vaidya and J. J. Rodrigues, "User-centric plug-and-play functionality for ipv6-enabled wireless sensor networks." IEEE Communications Society subject matter experts for publication, pp. 15,May, 2010

[12] O. Postolache, P. Girao, J. M. Dias Pereira, J. Pincho, C.Maia Moura, G. Postolache, "Smart walker for pervasive healthcare", Proceedings of International Conference on Sensing Technology (ICST), Parlmerston North, NZ, Dec. 2011, pp. 1-5.

[13] M. K. Hu, "Visual pattern recognition by moment invariants," Information Theory, IRE Transactions, vol. 8, pp. 179-187, 1962.

[14] O. Postolache, J. Freire, P. S. Girao, et al. "Smart sensor architecture for vital signs and motor activity monitoring of wheelchair'users.", IEEE 2012 Sixth International Conference on Sensing Technology, pp. 167172, December, 2012. 